\title{
New features of the layer-by-layer deposition of microcrystalline silicon films revealed by spectroscopic ellipsometry and high resolution transmission electron microscopy
}

\author{
P. Roca i Cabarrocas, ${ }^{\text {a) }}$ S. Hamma, and A. Hadjadj \\ Laboratoire de Physique des Interfaces et des Couches Minces (UPR 258 du CNRS), Ecole Polytechnique, \\ 91128 Palaiseau Cedex, France
}

\section{J. Bertomeu and J. Andreu}

Department de Física Aplicada i Electrònica, Universitat de Barcelona, Av. Diagonal 647, E-08028 Barcelona, Spain

(Received 26 March 1996; accepted for publication 24 May 1996)

\begin{abstract}
Spectroscopic ellipsometry and high resolution transmission electron microscopy have been used to characterize microcrystalline silicon films. We obtain an excellent agreement between the multilayer model used in the analysis of the optical data and the microscopy measurements. Moreover, thanks to the high resolution achieved in the microscopy measurements and to the improved optical models, two new features of the layer-by-layer deposition of microcrystalline silicon have been detected: (i) the microcrystalline films present large crystals extending from the $a-\mathrm{Si}: \mathrm{H}$ substrate to the film surface, despite the sequential process in the layer-by-layer deposition; and (ii) a porous layer exists between the amorphous silicon substrate and the microcrystalline silicon film. (C) 1996 American Institute of Physics. [S0003-6951(96)02730-1]
\end{abstract}

In situ ellipsometry is a powerful nondestructive technique providing detailed information on the growth mechanisms and optical properties of thin films. ${ }^{1}$ In order to get information on the composition of a film, the real $\left\langle\epsilon_{1}\right\rangle$ and imaginary $\left\langle\epsilon_{2}\right\rangle$ parts of the pseudodielectric function of the system film plus substrate, deduced from the ellipsometric angles $\Delta$ and $\Psi$, are compared to those of an optical model based on Bruggeman's effective-medium theory. ${ }^{2}$ The differences between the measured and calculated data are minimized by a linear regression analysis. In recent years we have applied this technique to study the layer-by-layer deposition of microcrystalline silicon films. ${ }^{3}$ In this method, microcrystalline silicon films are obtained by alternating the deposition of hydrogenated amorphous silicon $(a-\mathrm{Si}: \mathrm{H})$ during a time $T_{\mathrm{Si}}$ with its exposure to a hydrogen plasma during a time $T_{\mathrm{H}}$. The major conclusions deduced from these studies are: (i) the nucleation of a crystalline phase within the $a$-Si:H network takes place once the initially dense $a-\mathrm{Si}: \mathrm{H}$ film has been converted into porous $a-\mathrm{Si}: \mathrm{H}$ by the hydrogen plasma exposure, ${ }^{4}$ (ii) the crystallization of the $a$-Si:H film deposited during the time $T_{\mathrm{Si}}$ is related to the diffusion of hydrogen, leading to nanovoid and broken bond formation processes, ${ }^{5}$ and (iii) there is a substrate dependence of the long term evolution of the properties of the already deposited films. ${ }^{6}$

Because most of our previous results were based on in situ ellipsometry and because of the increasing complexity of optical models used to fit the experimental data, we have performed an independent validation of the optical models by high resolution transmission electron microscopy (HRTEM) measurements. An excellent agreement between the results of the optical models and the HRTEM measurements has been achieved, in agreement with previous reports. ${ }^{7}$ Moreover, the HRTEM measurements have allowed

${ }^{a)}$ Electronic mail: roca@ poly.polytechnique.fr us to improve the optical models and reveal new features of the layer-by-layer deposition of $\mu c-\mathrm{Si}$.

Microcrystalline silicon films were codeposited on different substrates by the layer-by-layer technique. Figure 1 shows the experimental and calculated real and imaginary parts of the pseudodielectric function of a system consisting of a $\mu c$-Si layer grown on an $a-\mathrm{Si}: \mathrm{H}$ film deposited on a crystalline silicon substrate. The $a$-Si:H film was obtained by the rf glow discharge decomposition of pure silane. After its exposure to air this film was loaded along with other substrates for the layer-by-layer deposition of a microcrystalline silicon layer. We have previously reported that the native oxyde layer on the $a$-Si:H substrate has no effect on the growth of the $\mu c-\mathrm{Si}$ film. ${ }^{6}$ The details of the deposition sys-

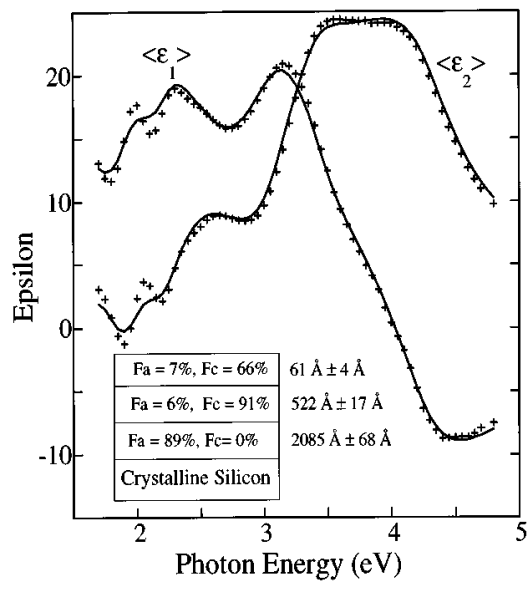

FIG. 1. Experimental (crosses) and simulated (solid line) real and imaginary parts of the pseudodielectric function of a microcrystalline silicon film deposited on an $a-\mathrm{Si}: \mathrm{H}$ film deposited on a crystalline substrate. A schematic view of the optical model used to fit the experimental data is shown in the figure. $F_{a}$ and $F_{c}$ represent, respectively, the amorphous and crystalline fractions, the difference to $100 \%$ corresponding to the void fraction. Note the poor quality of the fit in the low energy part of the spectra. 
tem have been published elsewhere. ${ }^{8}$ The $\mu c$-Si layer was obtained by repeating 50 cycles of $a-\mathrm{Si}: \mathrm{H}$ deposition during a time $T_{\mathrm{Si}}=20 \mathrm{~s}$ and exposure to a hydrogen plasma during a time $T_{\mathrm{H}}=40 \mathrm{~s}$. The ellipsometric angles were measured with a UVISEL spectroscopic phase modulated ellipsometer. ${ }^{9}$ In accordance with the deposition sequence, the optical model used to fit the experimental data consisted of a crystalline silicon substrate, an $a$-Si:H layer, a $\mu c$-Si layer, and a surface roughness layer. As shown in Fig. 1, the fit to the experimental data is excellent, except in the low energy part of the spectrum. This discrepancy suggests that the bottom part of the film is not well described by the present model. Indeed, this model gives a void fraction for the $a$-Si:H layer (11\%) much higher than expected (2\%) from the deposition conditions. Of course, it is possible to improve the optical model by adding more layers. However, without complementary measurements it would be difficult to say whether the improvement in the fit has any physical meaning.

In order to get complementary information on the structure of the film, the same sample was prepared in the following manner for transmission electron microscopy measurements: $2 \mathrm{~mm}$ wide stripes were cut with a diamond saw. Two stripes were bonded with the films facing each other with an M-BOND 610 glue, and again a series of $350 \mu \mathrm{m}$ thick stripes were cut with the same saw. These stripes were thinned down to $30 \mu \mathrm{m}$ with silicon carbide and aluminum oxide abrasive papers of decreasing grain size $(15,9,3,1$, and $0.3 \mu \mathrm{m})$. At this point the specimens are mounted on a copper grid and introduced into a Gatan 600 ion milling system which uses two $5 \mathrm{kV} \mathrm{Ar}^{+}$ion guns with an incidence angle of $15^{\circ}$ with respect to the surface. The ion current was $0.5 \mathrm{~mA}$ for each ion gun. The milling is stopped when a hole is detected by optical microscopy in the region of interest. The cross section of the samples was observed by high resolution transmission electron microscopy (HRTEM) in a Phillips CM30 microscope with an acceleration potential of 300 $\mathrm{kV}$.

Figure 2(a) shows a TEM micrograph of the whole film in which the crystalline substrate, the $a-\mathrm{Si}: \mathrm{H}$ layer and the $\mu c$-SI layer are clearly differentiated. In the $\mu c$-Si layer grains with lateral sizes up to $250 \AA$ and extending vertically from the $a$-Si:H substrate to the film surface are clearly observed. This columnar-like growth was not expected from the layer-by-layer process in which a 20 - $\AA$-thick $a$-Si:H layer is deposited at each cycle. Moreover, diffraction patterns taken at different points of the sample did not show any preferential orientation. Further work is necessary to understand why the crystal grains extend through the whole film thickness. Figures 2(b) and 2(c) show HRTEM images of the crystalline silicon $/ a-\mathrm{Si}: \mathrm{H}$ interface and of the $\mu c$-Si layer. While the $c-\mathrm{Si} / a-\mathrm{Si}: \mathrm{H}$ interface is abrupt, the $a-\mathrm{Si}: \mathrm{H} / \mu c-\mathrm{Si}$ interface is rough, the thickness of this interface being $\approx 100$ $\AA$ and comparable to the surface roughness of the $\mu c-\mathrm{Si}$ layer. The top part of Fig. 2(c) corresponds to the M-BOND 610 glue used to prepare the samples. The HRTEM observation of the $\mu c$-Si layer [Fig. 2(c)] suggests that this layer is completely crystallized, without any remaining amorphous phase. In this figure one can observe some fringes with a large separation corresponding to moiré interference

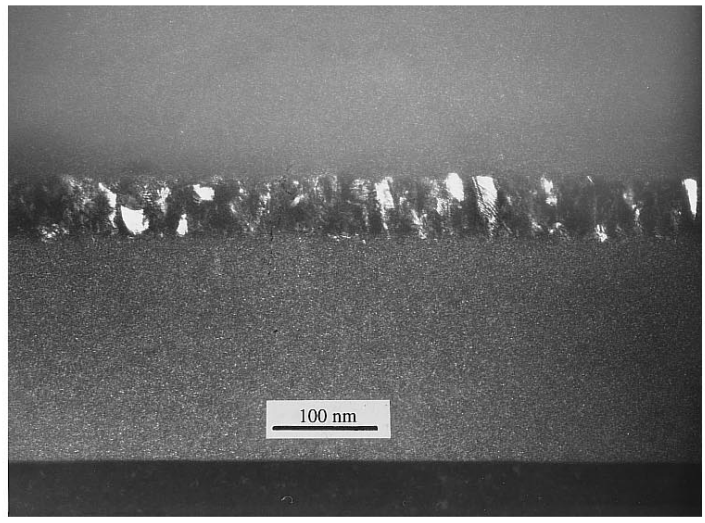

(a)

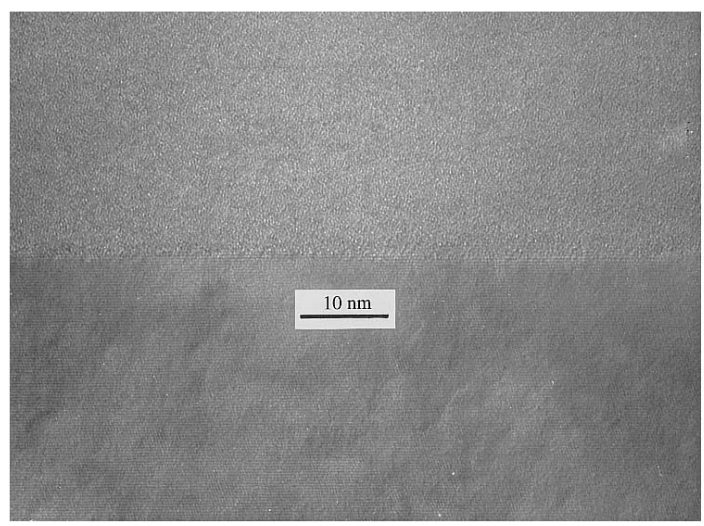

(b)

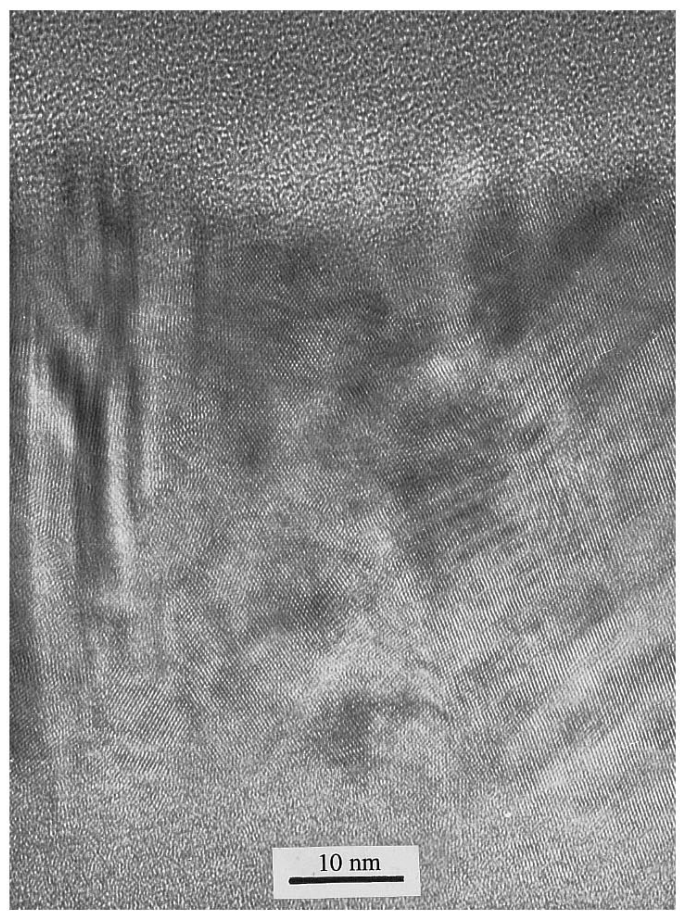

(c)

FIG. 2. High resolution transmission electron microscopy images of (a) a $\mu$ $c$-Si film deposited by the layer-by-layer technique on an $a$-Si:H film deposited on crystalline silicon; (b) the interface between the crystalline silicon and the $a$-Si:H film; and (c) the $\mu c$-Si film.

fringes ${ }^{10}$ produced by two crystals, one lying on top of the other with slightly different orientations. Moreover, the $\langle 1,1,1\rangle$ planes of $c$-Si can be clearly distinguished in Fig. 2(a) for the $c$-Si substrate. By using the expected distance be- 
tween these planes $(3.1356 \AA$ ) we have calibrated the thickness of the different layers with a precision better than $2.7 \%$. The following values have been deduced: $a$-Si:H layer 2000 $\AA$; $\mu c$-Si layer $550 \AA$; surface roughness and thickness of the $a$-Si:H/ $\mu c$-Si interface $\leqslant 100 \AA$. These values are in excellent agreement with these deduced from the optical model (Fig. 1), despite the poor quality of the fit in the low energy part of the spectra. The high crystalline fraction obtained from the SE model (Fig. 1) shows that the crystallites observed in the HRTEM images dominate the optical properties, and that the grain boundaries, where an amorphous tissue can be present, have very low influence.

Turning our attention to the SE models, the HRTEM pictures suggest that the $a-\mathrm{Si}: \mathrm{H} / \mu c-\mathrm{Si}$ interface should be included in the optical model. Therefore, we added a new layer in the optical model formed by a mixture of $a-\mathrm{Si}: \mathrm{H}$, $\mu c$-Si and voids, as is the case for the surface roughness layer. Indeed, adding a new layer improved the fit. However, when running the model to minimize the difference between the experimental and calculated values, it appeared that the essential feature of the interface layer was not its crystallinity but rather its higher porosity when compared to that of the underlying $a-\mathrm{Si}: \mathrm{H}$ or to that of the $\mu c$-Si layer. As suggested by the HRTEM pictures, we can force the presence of an interface layer which would be $40 \AA$ thick with a composition of $F_{a}=43 \%$ and $F_{c}=53 \%$. However, this interface layer does not improve the fit. This may result from the fact that its thickness is of the order of the precision of the ellipsometric models. The only way to improve the fit in the low energy part is the introduction of $a \approx 400 \AA$ thick $a$-Si:H layer having a porosity of $8 \%$. Figure 3 shows the same experimental data as Fig. 1, along with the results of the fit using the model schematically described in the lower part of the figure. We observe that in this case the low energy part of the spectra (interference fringes) is well described by the model. Moreover, contrary to the results of Fig. 1, the $a$-Si:H substrate is very dense, as expected from the deposition conditions. Therefore, the important results in Fig. 3 is the presence of the $400 \AA$ thick, $8 \%$ porous $a$-Si:H interface layer between the $a-\mathrm{Si}: \mathrm{H}$ and the $\mu c$-Si layers. Indeed, we have shown in our previous studies that in order to grow $\mu c-\mathrm{Si}$ on top of $a-\mathrm{Si}: \mathrm{H}$ it is necessary to form a highly porous $a-\mathrm{Si}: \mathrm{H}$ layer where crystal nucleation takes place. ${ }^{4}$ The results in Fig. 3 suggest that the porous interface layer is the relic of the highly porous layer formed by the hydrogen plasma during which the nucleation of crystallites took place.

In conclusion, we have shown that analysis of the SE measurements in the framework of the effective medium approximation gives detailed information on the thickness and composition of multilayer structures, in excellent agreement

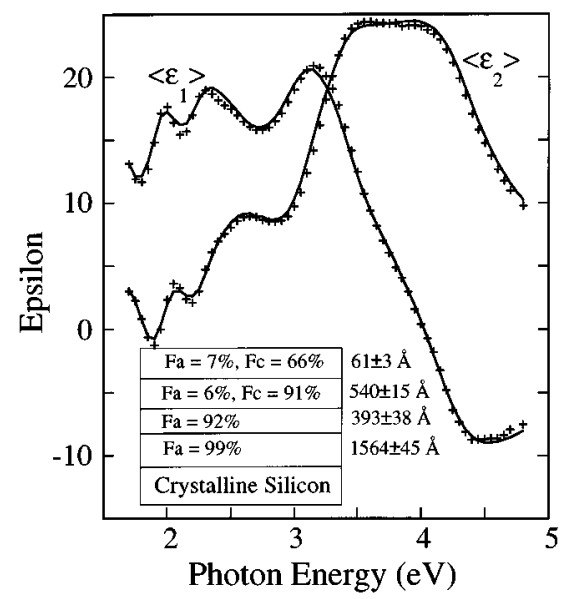

FIG. 3. Experimental (crosses) and simulated (solid line) real and imaginary parts of the pseudodielectric function of a microcrystalline silicon film deposited on an $a-\mathrm{Si}: \mathrm{H}$ film deposited on a crystalline substrate. The optical model used to fit the experimental data has been improved with respect to that shown in Fig. 1 by inserting an interface layer between the $a$-Si:H substrate and the $\mu c$-Si film, suggested by the micrographs shown in Fig. 2.

with high resolution transmission electron microscopy measurements. Moreover, the HRTEM measurements have revealed the formation of large crystals by layer-by-layer deposition of $\mu c-\mathrm{Si}$, while refinement of the optical models confirms the importance of the formation of a porous phase in the nucleation of crystallites.

The authors acknowledge the collaboration of ScientificTechnical Services of the Universitat de Barcelona, where HRTEM measurements were performed. The contribution of the Universitat de Barcelona was supported by DGICYT of the Spanish Government under Program MAT94-0262, while the work at Ecole Polytechnique was partly supported by a JOULE II Contract No. CEE JOU2.CT 940403.

${ }^{1}$ B. Drévillon, Prog. Cryst. Growth Characterization Mater. 27, 1 (1993).

${ }^{2}$ A. G. Bruggeman, Ann. Phys. Leipzig 24, 636 (1935).

${ }^{3}$ N. Layadi, P. Roca i Cabarrocas, B. Drévillon, and I. Solomon, Phys. Rev. B 52, 5136 (1995).

${ }^{4}$ P. Roca i Cabarrocas, N. Layadi, B. Drévillon, and I. Solomon, J. NonCryst. Solids 198-200, 871 (1996).

${ }^{5}$ C. Godet, N. Layadi, and P. Roca i Cabarrocas, Appl. Phys. Lett. 66, 3146 (1995).

${ }^{6}$ P. Roca i Cabarrocas, N. Layadi, T. Heitz, and B. Drévillon, Appl. Phys. Lett. 66, 3609 (1995).

${ }^{7}$ K. Vedam, P. J. McMarr, and J. Narayan, Appl. Phys. Lett. 47, 339 (1985).

${ }^{8}$ P. Roca i Cabarrocas, J. Huc, A. Lloret, J. Y. Parey, and J. P. M. Schmitt, J. Vac. Sci. Technol. A 9, 2331 (1991).

${ }^{9}$ B. Drévillon, J. Y. Parey, M. Stchakovsky, R. Benferhat, Y. Josserand, and B. Schlayen, Proc. SPIE 1188, 174 (1990).

${ }^{10}$ L. Reimer, Transmission Electron Microscopy, Physics of Image Formation and Microanalysis, 2nd ed. (Springer, Berlin, 1989). 